\title{
Joint Distributions of Permutation Statistics and the Parabolic Cylinder Functions
}

\author{
Amy M. Fu' ${ }^{1}$ and Frank Z.K. Li $^{2}$ \\ ${ }^{1}$ School of Mathematics, \\ Shanghai University of Finance and Economics, \\ Shanghai 200433, P.R. China \\ ${ }^{2}$ Center for Combinatorics, LPMC, \\ Nankai University, Tianjin 300071, P.R. China \\ Emails: ${ }^{1}$ fu.mei@mail.shufe.edu.cn, ${ }^{2}$ zhkli@mail.nankai.edu.cn
}

\begin{abstract}
In this paper, we introduce a context-free grammar $G: x \rightarrow x y, y \rightarrow z u, z \rightarrow$ $z w, w \rightarrow x v, u \rightarrow x y z^{-1} v, v \rightarrow x^{-1} z w u$ over the variable set $V=\{x, y, z, w, u, v\}$. We use this grammar to study joint distributions of several permutation statistics related to descents, rises, peaks and valleys. By considering the pattern of an exterior peak, we introduce the exterior peaks of pattern 132 and of pattern 231. Similarly, peaks can also be classified according to their patterns. Let $D$ be the formal derivative operator with respect to the grammar $G$. By using a grammatical labeling, we show that $D^{n}(z)$ is the generating function of the number of permutations on $[n]=\{1,2, \ldots, n\}$ with given numbers of exterior peaks of pattern 132 and of pattern 231, and proper double descents. By solving a cylinder differential equation, we obtain an explicit formula of the generating function of $D^{n}(z)$, which can be viewed as a unification of the results of Elizalde-Noy, Barry, Basset, Fu and Gessel. Specializations lead to the joint distributions of certain consecutive patterns in permutations, as studied by Elizalde-Noy and Kitaev. By a different labeling with respect to the same grammar $G$, we derive the joint distribution of peaks of pattern 132 and of pattern 231, double descents and double rises, with the generating function also expressed by the parabolic cylinder functions. This formula serves as a refinement of the work of Carlitz-Scoville. Furthermore, we obtain the joint distribution of exterior peaks of pattern 132 and of pattern 231 over alternating permutations.
\end{abstract}

Keywords: grammatical labeling; context-free grammar; permutation statistics; generating function; the parabolic cylinder function.

AMS Classification: 05A15, 05A19.

\section{Introduction}

In this paper, we introduce a refinement of the exterior peaks based on their patterns. More precisely, an exterior peak of a permutation is either of pattern 132 or 231. Similarly, we may define the types of a peak of a permutation. By giving a context-free grammar, we study the joint distribution of exterior peaks of pattern 132 and of pattern 231, and proper double descents over permutations on $[n]=\{1,2, \ldots, n\}$. In the same manner, we study the joint distribution of peaks of pattern 132 and of pattern 231, double descents and double rises on permutations. It turns out that the generating functions for the joint distributions are in connection with the parabolic cylinder functions. 
The notion of exterior peaks was introduced by Aguiar, Bergeron and Nymanin [2]. Let $\pi=\pi_{1} \pi_{2} \cdots \pi_{n}$ be a permutation on $[n]$ and $\pi_{0}=0$, then for the index $1 \leq i \leq n-1$, we call $i$ an exterior peak if $\pi_{i-1}<\pi_{i}>\pi_{i+1}$. For example, the permutation 534621 has two exterior peaks 1 and 4 . For $n \geq 0$, denote by $T(n, k)$ the number of permutations on $[n]$ with $k$ exterior peaks and let

$$
T_{n}(x)=\sum_{k \geq 0} T(n, k) x^{k} .
$$

Gessel [20, A008971] obtained the generating function of $T_{n}(x)$.

Theorem 1.1 (Gessel [20]) We have

$$
\sum_{n=0}^{\infty} T_{n}(x) \frac{t^{n}}{n !}=\frac{\sqrt{1-x}}{\sqrt{1-x} \cosh (\sqrt{1-x} t)-\sinh (\sqrt{1-x} t)}
$$

Given a permutation $\pi=\pi_{1} \pi_{2} \cdots \pi_{n}$ on $[n]$, for $2 \leq i \leq n-1$, an index $i$ is a proper double descent if $\pi_{i-1}>\pi_{i}>\pi_{i+1}$. As an example, the permutation 653421 has two proper double descents 2 and 5 . For $n \geq 0$, let $U(n, k)$ be the number of permutations on $[n]$ with $k$ proper double descents and let

$$
U_{n}(y)=\sum_{k \geq 0} U(n, k) y^{k} .
$$

By solving a second-order ordinary differential equation, Elizalde and Noy [9] obtained the generating function of $U_{n}(y)$.

Theorem 1.2 (Elizalde-Noy [9]) We have

$$
\sum_{n=0}^{\infty} U_{n}(y) \frac{t^{n}}{n !}=\frac{2 \sqrt{(y-1)(y+3)} e^{(1-y+\sqrt{(y-1)(y+3)})} \frac{t}{2}}{1+y+\sqrt{(y-1)(y+3)}-(1+y-\sqrt{(y-1)(y+3)}) e^{\sqrt{(y-1)(y+3)} t}} .
$$

Barry [3] and Basset [4] independently derived the generating function of permutations on $[n]$ containing no proper double descents.

Theorem 1.3 (Elizalde-Noy [9], Barry [3], Basset [4]) We have

$$
\sum_{n=0}^{\infty} U(n, 0) \frac{t^{n}}{n !}=\frac{\sqrt{3}}{2} \frac{e^{\frac{t}{2}}}{\cos \left(\frac{\sqrt{3} t}{2}+\frac{\pi}{6}\right)}
$$

The first named author [10] introduced the context-free grammar

$$
x \rightarrow x y, y \rightarrow x z, z \rightarrow z w, w \rightarrow x z,
$$

and obtained the joint distribution of exterior peaks and proper double descents over permutations on $[n]$. For $n \geq 0$, let

$$
P_{n}(x, y, z, w)=\sum_{\pi \in \mathfrak{S}_{n}} x^{e p(\pi)} y^{p d d(\pi)} z^{e p(\pi)+1} w^{n-2 e p(\pi)-p d d(\pi)},
$$

where $\mathfrak{S}_{n}$ is the set of permutations on $[n], e p(\pi)$ and $p d d(\pi)$ are the number of exterior peaks and the number of proper double descents in permutation $\pi$, respectively. 
Theorem 1.4 (Fu [10]) We have

$$
\sum_{n=0}^{\infty} P_{n}(x, y, z, w) \frac{t^{n}}{n !}=\frac{2 z \sqrt{(y+w)^{2}-4 x z} e^{\frac{t}{2}\left(w-y+\sqrt{(y+w)^{2}-4 x z}\right)}}{y+w+\sqrt{(y+w)^{2}-4 x z}-\left(y+w-\sqrt{(y+w)^{2}-4 x z}\right) e^{t \sqrt{(y+w)^{2}-4 x z}}} .
$$

Notice that Theorem 1.4 reduces to Theorem 1.1, Theorem 1.2 and Theorem 1.3.

In this paper, we give a refinement of the generating function $P_{n}(x, y, z, w)$ by considering the pattern of an exterior peak. For a permutation $\pi=\pi_{1} \pi_{2} \cdots \pi_{n}$ and $1 \leq i \leq n-1$, if $i$ is an exterior peak with $\pi_{i-1}<\pi_{i}>\pi_{i+1}$, then we say $i$ is an exterior peak of pattern 132 if $\pi_{i-1}<\pi_{i+1}$, or an exterior peak of pattern 231 if $\pi_{i-1}>\pi_{i+1}$. For example, the permutation 534621 has one index 1 as an exterior peak of pattern 132, and one index 4 as an exterior peak of pattern 231.

For $n \geq 0$, let $P_{n}(i, j, k)$ be the number of permutations on $[n]$ with $i$ exterior peaks of pattern $132, j$ exterior peaks of pattern 231, and $k$ proper double descents. Define

$$
P_{n}(x, y, z, w, u, v)=\sum_{i, j, k=0}^{n} P_{n}(i, j, k) x^{i} v^{i} u^{j} z^{j+1} y^{k} w^{n-2 i-2 j-k}
$$

or equivalently,

$$
P_{n}(x, y, z, w, u, v)=\sum_{\pi \in \mathfrak{S}_{n}} x^{e p_{1}(\pi)} v^{e p_{1}(\pi)} u^{e p_{2}(\pi)} z^{e p_{2}(\pi)+1} y^{p d d(\pi)} w^{n-2\left(e p_{1}(\pi)+e p_{2}(\pi)\right)-p d d(\pi)},
$$

where $e p_{1}(\pi)$ and $e p_{2}(\pi)$ are the numbers of exterior peaks of pattern 132 and of pattern 231 in the permutation $\pi$, respectively. We introduce the context-free grammar on the variable set $V=\{x, y, z, w, u, v\}$ :

$$
G: x \rightarrow x y, y \rightarrow z u, z \rightarrow z w, w \rightarrow x v, u \rightarrow x y z^{-1} v, v \rightarrow x^{-1} z w u
$$

which can be used to derive an explicit formula for the generating function of $P_{n}(x, y, z, w, u, v)$ in terms of the parabolic cylinder functions.

Let us recall some basic knowledges of the parabolic cylinder functions, where one can refer to $[17,16,18,1]$ for more details. Let $n$ be a nonnegative integer, then we adapt the notation of the shifted factorial as follows:

$$
\begin{aligned}
& (a)_{0}=1, \\
& (a)_{n}=a(a+1) \cdots(a+n-1), \quad n>0 .
\end{aligned}
$$

The confluent hypergeometric function ${ }_{1} F_{1}$ with parameters $a, b$ and $z$ is defined as

$$
{ }_{1} F_{1}(a ; b ; z)=\sum_{n=0}^{\infty} \frac{(a)_{n}}{(b)_{n}} \frac{z^{n}}{n !} .
$$

The parabolic cylinder function $D_{a}(z)$ introduced by Whittaker and Watson $[18, \S 16.5]$ is defined as

$$
D_{a}(z)=2^{\frac{a}{2}} \sqrt{\pi} e^{-\frac{z^{2}}{4}}\left(\frac{1}{\Gamma\left(\frac{1-a}{2}\right)}{ }_{1} F_{1}\left(-\frac{a}{2} ; \frac{1}{2} ; \frac{z^{2}}{2}\right)-\frac{\sqrt{2} z}{\Gamma\left(-\frac{a}{2}\right)}{ }_{1} F_{1}\left(\frac{1-a}{2} ; \frac{3}{2} ; \frac{z^{2}}{2}\right)\right) .
$$


Theorem 1.5 Let $\delta=\sqrt{x v-z u}$ and $\hat{\delta}=\sqrt{z u-x v}$. We have

$$
\sum_{n=0}^{\infty} P_{n}(x, y, z, w, u, v) \frac{t^{n}}{n !}=\frac{z(p q(w-y)+(\hat{\delta} p s-\delta q r)) e^{\frac{w-y}{2} t+\frac{\delta^{2}}{4} t^{2}}}{(\hat{\delta} s-q y) D_{\frac{z u-y w}{\delta^{2}}}\left(\delta t+\frac{w-y}{\delta}\right)+(p w-\delta r) D_{\frac{x v-y w}{\delta^{2}}}\left(\hat{\delta} t+\frac{y-w}{\hat{\delta}}\right)}
$$

where

$$
\begin{aligned}
& p=D_{\frac{z u-y w}{\delta^{2}}}\left(\frac{w-y}{\delta}\right), \quad q=D_{\frac{x v-y w}{\delta^{2}}}\left(\frac{y-w}{\hat{\delta}}\right), \\
& r=D_{\frac{x v-y w}{\delta^{2}}}\left(\frac{w-y}{\delta}\right), \quad s=D_{\frac{z u-y w}{\delta^{2}}}\left(\frac{y-w}{\hat{\delta}}\right) .
\end{aligned}
$$

It can be seen that Theorem 1.5 is a refinement of Theorem 1.4. By specializing the variables in Theorem 1.5, we obtain the distribution of the total number of consecutive patterns 231 and 321 in permutations on $[n]$. For $n \geq 0$, let $L(n, k)$ be the number of permutations on $[n]$ with $k$ consecutive patterns 231 and 321 , and let

$$
L_{n}(x)=\sum_{k \geq 0} L(n, k) x^{k}
$$

Theorem 1.6 We have

$$
\sum_{n=0}^{\infty} L_{n}(x) \frac{t^{n}}{n !}=\frac{e^{\frac{t(t+2)(1-x)}{2}}}{1+x e^{\frac{x-1}{2}} \int_{t+1}^{1} e^{\frac{1-x}{2} s^{2}} d s}
$$

As another specialization of Theorem 1.5, we obtain the joint distribution of exterior peaks of pattern 132 and of pattern 231 over permutations on $[n]$. For $n \geq 0$, denote by $T(n, i, j)$ the number of permutations on $[n]$ with $i$ exterior peaks of pattern 132 and $j$ exterior peaks of pattern 231, and let

$$
T_{n}(x, y)=\sum_{i, j \geq 0} T(n, i, j) x^{i} y^{j}
$$

Theorem 1.7 We have

$$
\sum_{n=0}^{\infty} T_{n}(x, y) \frac{t^{n}}{n !}=\frac{e^{\frac{x-y}{2} t^{2}}}{{ }_{1} F_{1}\left(\frac{1-y}{2(x-y)} ; \frac{1}{2} ; \frac{x-y}{2} t^{2}\right)-t_{1} F_{1}\left(\frac{1}{2}+\frac{1-y}{2(x-y)} ; \frac{3}{2} ; \frac{x-y}{2} t^{2}\right)} .
$$

Specializations of Theorem 1.7 yield the generating function of the number of permutations on $[n]$ with no exterior peak of pattern 132 and the generating function of the number of permutations on $[n]$ avoiding the consecutive pattern 231 due to Kitaev $[12,13]$, as well as the distribution of consecutive patterns 231 in permutations on $[n]$ given by Elizalde and Noy [9].

Meanwhile, by using the context-free grammar $G$, we can derive the joint distribution of more permutation statistics. Given a permutation $\pi=\pi_{1} \pi_{2} \cdots \pi_{n}$ on $[n]$, first set $\pi_{0}=\pi_{n+1}=$ 0 . Following the terminology in $[15, \S 1.5]$, for $1 \leq i \leq n$, we call an index $i$ a peak (or a 
maxima [5], or a modified maximum [11]) if $\pi_{i-1}<\pi_{i}>\pi_{i+1}$, a valley if $\pi_{i-1}>\pi_{i}<\pi_{i+1}$, a double rise if $\pi_{i-1}<\pi_{i}<\pi_{i+1}$, or a double descent if $\pi_{i-1}>\pi_{i}>\pi_{i+1}$. For example, the permutation 4356721 has two peaks 1 and 5, one valley 2, two double rises 3 and 4, and two double descents 6 and 7 . Denote by $p(\pi), v(\pi), d d(\pi)$ and $d r(\pi)$ the numbers of peaks, valleys, double descents and double rises in permutation $\pi$, respectively. Carlitz and Scoville [5] studied the joint distribution of peaks, valleys, double rises and double descents over permutations on $[n]$. For $n \geq 1$, define

$$
F_{n}(x, y, z, w)=\sum_{\pi \in \mathfrak{S}_{n}} x^{p(\pi)-1} z^{v(\pi)} y^{d d(\pi)} w^{d r(\pi)}
$$

They obtained the following generating function of $F_{n}(x, y, z, w)$, see also [11, Exercise 3.3.46] and [15, Exercise 1.61].

Theorem 1.8 (Carlitz-Scoville [5]) We have

$$
\sum_{n=1}^{\infty} F_{n}(x, y, z, w) \frac{t^{n}}{n !}=\frac{e^{\beta t}-e^{\alpha t}}{\beta e^{\alpha t}-\alpha e^{\beta t}}
$$

where $\alpha \beta=x z$ and $\alpha+\beta=y+w$.

We also obtain a refinement of the generating function $F_{n}(x, y, z, w)$ by considering the patterns of peaks. Given a permutation $\pi=\pi_{1} \pi_{2} \cdots \pi_{n}$ and $1 \leq i \leq n$, if $i$ is a peak with $\pi_{i-1}<\pi_{i}>\pi_{i+1}$, then we call $i$ a peak of pattern 132 if $\pi_{i-1} \leq \pi_{i+1}$, or a peak of pattern 231 if $\pi_{i-1}>\pi_{i+1}$. Note that only when $\pi=1$ a peak $i$ can be of pattern 132 with $\pi_{i-1}=\pi_{i+1}$. For $n \geq 1$, let $Q_{n}(i, j, k, \ell)$ be the number of permutations on $[n]$ with $i$ peaks of pattern 132 , $j$ peaks of pattern 231, $k$ double descents and $\ell$ double rises. For $n \geq 1$, define

$$
Q_{n}(x, y, z, w, u, v)=\sum_{i, j, k, \ell=0}^{n} Q_{n}(i, j, k, \ell) x^{i} v^{i} u^{j} z^{j} y^{k} w^{\ell},
$$

or equivalently,

$$
Q_{n}(x, y, z, w, u, v)=\sum_{\pi \in \mathfrak{S}_{n}} x^{p_{1}(\pi)} v^{p_{1}(\pi)} u^{p_{2}(\pi)} z^{p_{2}(\pi)} y^{d d(\pi)} w^{d r(\pi)},
$$

where $p_{1}(\pi)$ is the number of peaks of pattern 132 in permutation $\pi$ and $p_{2}(\pi)$ is the number of peaks of pattern 231 in permutation $\pi$. Based on the same context-free grammar $G(1.5)$, we derive the generating function of $Q_{n}(x, y, z, w, u, v)$.

Theorem 1.9 Set $Q_{0}(x, y, z, w, u, v)=w$. Let $\delta, \hat{\delta}, p, q, r, s$ be defined as in Theorem 1.5. Then

$$
\begin{aligned}
\sum_{n=0}^{\infty} Q_{n}(x, y, z, w, u, v) \frac{t^{n}}{n !} & \\
& =\frac{\left(\delta^{2} t+w-y\right)(p w-\delta r) D_{\frac{x v-y w}{\delta^{2}}}\left(\hat{\delta} t+\frac{y-w}{\hat{\delta}}\right)}{(\hat{\delta} s-q y) D_{\frac{z u-y w}{\delta^{2}}}\left(\delta t+\frac{w-y}{\delta}\right)+(p w-\delta r) D_{\frac{x v-y w}{\delta^{2}}}\left(\hat{\delta} t+\frac{y-w}{\hat{\delta}}\right)}
\end{aligned}
$$




$$
+\frac{\delta(\hat{\delta} s-q y) D_{\frac{x v-y w}{\delta^{2}}}\left(\delta t+\frac{w-y}{\delta}\right)+\hat{\delta}(p w-\delta r) D_{\frac{z u-y w}{\delta^{2}}}\left(\hat{\delta} t+\frac{y-w}{\hat{\delta}}\right)}{(\hat{\delta} s-q y) D_{\frac{z u-y w}{\delta^{2}}}\left(\delta t+\frac{w-y}{\delta}\right)+(p w-\delta r) D_{\frac{x v-y w}{\delta^{2}}}\left(\hat{\delta} t+\frac{y-w}{\hat{\delta}}\right)} .
$$

Note that Theorem 1.9 can be viewed as a refinement of Theorem 1.8. Combining Theorem 1.5 and Theorem 1.9, we are led to the joint distribution of exterior peaks of pattern 132 and of pattern 231 over alternating permutations on $[n]$. For $n \geq 0$, let $T^{A}(n, i, j)$ be the number of alternating permutations on $[n]$ with $i$ exterior peaks of pattern 132 and $j$ exterior peaks of pattern 231, and let

$$
T_{n}^{A}(x, y)=\sum_{i, j \geq 0} T^{A}(n, i, j) x^{i} y^{j}
$$

Theorem 1.10 We have

$$
\sum_{n=0}^{\infty} T_{n}^{A}(x, y) \frac{t^{n}}{n !}=\frac{e^{\frac{x-y}{2} t^{2}}\left(1+t_{1} F_{1}\left(\frac{x}{2(x-y)} ; \frac{3}{2} ;-\frac{x-y}{2} t^{2}\right)\right)}{{ }_{1} F_{1}\left(-\frac{y}{2(x-y)} ; \frac{1}{2} ; \frac{x-y}{2} t^{2}\right)} .
$$

The rest of this paper is organized as follows. In Section 2, we give an overview of the formal derivative with respect to a context-free grammar, and provide grammatical labelings on permutations to generate the polynomials $P_{n}(x, y, z, w, u, v)$ and $Q_{n}(x, y, z, w, u, v)$. In Section 3, we give proofs of Theorem 1.5 and Theorem 1.9 by establishing a parabolic cylinder differential equation. Then we show that Theorem 1.4 and Theorem 1.8 are specializations of Theorem 1.5 and Theorem 1.9. Section 4 is devoted to the proofs of Theorem 1.6, Theorem 1.7 and Theorem 1.10 .

\section{Grammatical labelings}

In this section, we first recall some basic backgrounds of the formal derivative with respect a context-free grammar and the grammatical labeling. Then with the context-free grammar $G$ (1.5), we give the corresponding grammatical labeling to derive the generating function $P_{n}(x, y, z, w, u, v)$. By using a different grammatical labeling based on the same grammar $G$ (1.5), we derive the generating function $Q_{n}(x, y, z, w, u, v)$.

Let $V$ be a variable set. A context-free grammar $G$ is defined as a set of substitution rules replacing a variable in $V$ by a Laurent polynomial of variables in $V$. For variables $u, v \in V$, define a linear operator $D$ with respect to $G$ with the following properties:

(i) $D(u+v)=D(u)+D(v)$;

(ii) $D(u v)=D(u) v+u D(v)$;

(iii) $D(c)=0$, if $c$ is a constant. 
Thus, for integer $n \geq 0$, the operator $D$ satisfies the Leibniz rule

$$
D^{n}(u v)=\sum_{k=0}^{n}\left(\begin{array}{l}
n \\
k
\end{array}\right) D^{k}(u) D^{n-k}(v) .
$$

For a Laurent polynomial $w$ of variables in $V$, we define the generating function of $w$ as

$$
\operatorname{Gen}(w, t)=\sum_{n=0}^{\infty} D^{n}(w) \frac{t^{n}}{n !}
$$

Then due to (2.1) and (2.2), the following relations hold:

$$
\begin{aligned}
\operatorname{Gen}(u v, t) & =\operatorname{Gen}(u, t) \operatorname{Gen}(v, t), \\
\operatorname{Gen}^{\prime}(u, t) & =\operatorname{Gen}(D(u), t),
\end{aligned}
$$

where $u, v$ are Laurent polynomials of variables in $V$, and $\operatorname{Gen}^{\prime}(u, t)$ is the normal derivative with respect to $t$. We call the above operator $D$ the formal derivative with respect to the grammar $G$.

The idea of using the formal derivative with respect to a context-free grammar to study combinatorial structures was initiated by Chen [6]. Dumont [8] later found grammars for several classical combinatorial structures. For example, Dumont defined a context-free grammar

$$
x \rightarrow x y, y \rightarrow x y
$$

and used the corresponding formal derivative to generate the Eulerian polynomials $A_{n}(x)$, namely, $\left.D^{n}(x)\right|_{y=1}=x A_{n}(x)$.

The concept of the grammatical labeling was introduced in [7] to build the connections between context-free grammars and the combinatorial structures. A grammatical labeling is an assignment of the underlying elements of a combinatorial structure with constants or variables, which is consistent with the substitution rules of a context-free grammar $G$. For example, by using the grammar

$$
x \rightarrow x y, y \rightarrow x^{2},
$$

we may label the elements of a permutation $\pi$ on $[n]$ by assigning $\pi_{i}$ and $\pi_{i+1}$ both label $x$ if $i$ is an exterior peak and assigning all other elements label $y$. By this grammatical labeling, one can prove that $D^{n}(x)$ is the generating function of the number of permutations on $[n]$ with a given number of exterior peaks. This example can be found in [7], and see [14] for a similar example but related to the normal derivative given by Ma. In [10], by the corresponding grammatical labeling consistent with the grammar (1.1):

$$
x \rightarrow x y, y \rightarrow x z, z \rightarrow z w, w \rightarrow x z
$$

the first named author gave the generating function of $P_{n}(x, y, z, w)$.

Recall that $P_{n}(x, y, z, w, u, v)$ is defined in (1.3), that is,

$$
P_{n}(x, y, z, w, u, v)=\sum_{i, j, k=0}^{n} P_{n}(i, j, k) x^{i} v^{i} u^{j} z^{j+1} y^{k} w^{n-2 i-2 j-k} .
$$


Let $G$ be the context-free grammar over the variable set $V=\{x, y, z, w, u, v\}$ defined as in (1.5):

$$
G: x \rightarrow x y, y \rightarrow z u, z \rightarrow z w, w \rightarrow x v, u \rightarrow x y z^{-1} v, v \rightarrow x^{-1} z w u .
$$

Notice that the grammar $G$ is a refinement of grammars (2.5), (2.6) and (1.1). More precisely, substituting $w, u$ by $x$ and $z, v$ by $y$ in the grammar $G$ reduces to the grammar (2.5); substituting $z, u, v$ by $x$ and $w$ by $y$ in the grammar $G$ reduces to the grammar (2.6); and substituting $v$ by $z$ and $u$ by $x$ in the grammar $G$ reduces to the grammar (1.1).

Theorem 2.1 Let $D$ be the formal derivative with respect to the grammar $G(1.5)$. For $n \geq 0$, we have

$$
D^{n}(z)=P_{n}(x, y, z, w, u, v)
$$

The theorem asserts that the generating function $P_{n}(x, y, z, w, u, v)$ can be grammatically acquired by computing the formal derivatives of $z$ with respect to the grammar $G(1.5)$. For instance,

$$
D^{4}(z)=6 x z w^{2} v+5 z^{2} w^{2} u+5 x y z w v+y z^{2} w u+x y^{2} z v+3 x^{2} z v^{2}+2 x z^{2} u v+z w^{4} .
$$

Since the coefficient of $x y z w v$ in $D^{4}(z)$ is 5 , we can deduce that there are five permutations on $\{1,2,3,4\}$ with one exterior peak of pattern 132 and one proper double descent. It is easy to check that they are 2431, 1421, 4213, 4312 and 3214.

To prove the theorem, given a permutation $\pi=\pi_{1} \pi_{2} \cdots \pi_{n}$ on $[n]$, we assign a labeling of $\pi$ as follows. We first add an element 0 at the end of $\pi$ and label it by $z$. Then for $1 \leq i \leq n-1$, if $i$ is an exterior peak of pattern 132, label $\pi_{i}$ by $x$ and $\pi_{i+1}$ by $v$, if $i$ is an exterior peak of pattern 231, label $\pi_{i}$ by $u$ and $\pi_{i+1}$ by $z$, if $i$ is a proper double descent, label $\pi_{i+1}$ by $y$. The other elements of $\pi$ are all assigned label $w$. Denote by $w(\pi)$ the weight of permutation $\pi$ as the product of all labels of elements of $\pi$, i.e.,

$$
w(\pi)=x^{e p_{1}(\pi)} v^{e p_{1}(\pi)} u^{e p_{2}(\pi)} z^{e p_{2}(\pi)+1} y^{p d d(\pi)} w^{n-2\left(e p_{1}(\pi)+e p_{2}(\pi)\right)-p d d(\pi)} .
$$

Example 2.1 Let $\pi=534621$. The labeling of $\pi$ is as follows:

$$
\begin{array}{lllllll}
5 & 3 & 4 & 6 & 2 & 1 & 0 \\
x & v & w & u & z & y & z
\end{array}
$$

and $w(\pi)=x y z^{2} w u v$.

Proof of Theorem 2.1. We proceed to prove by induction on $n$. By (1.4) and (2.7), we observe that

$$
P_{n}(x, y, z, w, u, v)=\sum_{\pi \in \mathfrak{S}_{n}} w(\pi) .
$$

For $n=0$, the grammatical labeling of the empty permutation is given by 
which leads to $P_{0}(x, y, z, w, u, v)=z$. From the view of the formal derivative, we see that $D^{0}(z)=z$. Thus the theorem holds when $n=0$. Suppose that the theorem is valid for $n \geq 1$, that is, $D^{n}(z)=\sum_{\pi \in \mathfrak{S}_{n}} w(\pi)$.

Let $\pi=\pi_{1} \pi_{2} \ldots \pi_{n}$ be a permutation on $[n]$ with $i$ exterior peaks of pattern $132, j$ exterior peaks of pattern 231, and $k$ proper double descents. Hence the weight of $\pi$ is $x^{i} v^{i} u^{j} z^{j+1} y^{k} w^{n-2 i-2 j-k}$. To complete the proof for the case $n+1$, we first add an element 0 at the end of $\pi$, then generate permutations on $[n+1]$ by inserting $n+1$ before each element of $\pi$ (including the element 0 ). Depending on the position $n+1$ inserted, we can label $n+1$ by $x, u$ or $w$, and adjust labels of some related elements. There are six cases as follows.

Case 1. Insert $n+1$ before some element $\pi_{\ell}$ labeled by $x$. As shown below, the position of $n+1$ becomes an exterior peak of pattern 132 since $\pi_{\ell-1}<\pi_{\ell}$, so that we label $n+1$ by $x$ and relabel $\pi_{\ell}$ by $v$. The position of $\pi_{\ell}$ becomes a proper double descent since $n+1>\pi_{\ell}>\pi_{\ell+1}$, so we relabel $\pi_{\ell+1}$ by $y$.

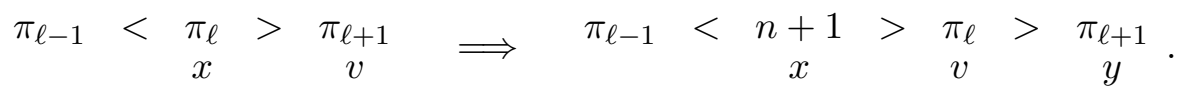

For example, inserting 7 before the element 5 in (2.8) yields the grammatical labeling:

$$
\begin{array}{llllllll}
7 & 5 & 3 & 4 & 6 & 2 & 1 & 0 \\
x & v & y & w & u & z & y & z
\end{array} .
$$

Therefore, this insertion of $n+1$ corresponds to the substitution rule $x \rightarrow x y$ in grammar $G$ (1.5). Since there are $i$ exterior peaks of pattern 132 in $\pi$, the insertion produces $i$ permutation on $[n+1]$ with $i$ exterior peaks of pattern 132, $j$ exterior peaks of pattern 231 , and $k+1$ proper double descents. The total weight of these $i$ permutations is

$$
i x^{i} v^{i} u^{j} z^{j+1} y^{k+1} w^{n-2 i-2 j-k} .
$$

Case 2. Insert $n+1$ before some element $\pi_{\ell}$ labeled by $v$. We label $n+1$ by $u$ and relabel $\pi_{\ell}$ by $z$ since the position of $n+1$ is an exterior peak of pattern 231 due to $\pi_{\ell-1}>\pi_{\ell}$, in addition, we relabel $\pi_{\ell-1}$ by $w$ since $\pi_{\ell-1}<n+1$, as illustrated below:

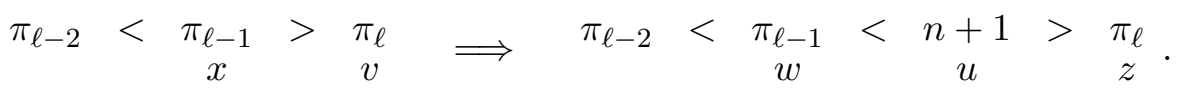

For example, if we insert 7 before element 3 in (2.8), then the labeling is changed to

$\begin{array}{cccccccc}5 & 7 & 3 & 4 & 6 & 2 & 1 & 0 \\ w & u & z & w & u & z & y & z\end{array}$.

Note that this insertion corresponds to the rule $v \rightarrow x^{-1} z w u$, and generates $i$ permutations on $[n+1]$ with $i-1$ exterior peaks of pattern $132, j+1$ exterior peaks of pattern 231 , and $k+1$ proper double descent. The total weight of these $i$ permutations is

$$
i x^{i-1} v^{i-1} u^{j+1} z^{j+2} y^{k+1} w^{n-2 i-2 j-k} .
$$


Case 3. Insert $n+1$ before some element $\pi_{\ell}$ with label $u$. Then the position of $n+1$ becomes an exterior peak of pattern 132 and the position of $\pi_{\ell}$ becomes a proper double descent, so that we label $n+1$ by $x$, and relabel $\pi_{\ell}$ by $v$ and $\pi_{\ell+1}$ by $y$ :

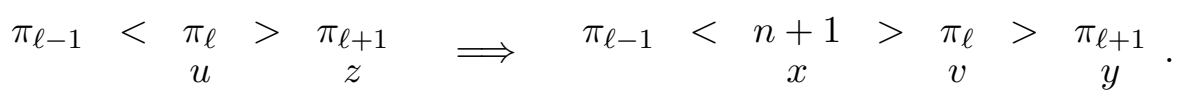

For example, we insert 7 before the element 6 in (2.8). Then we obtain

$\begin{array}{llllllll}5 & 3 & 4 & 7 & 6 & 2 & 1 & 0 \\ x & v & w & x & v & y & y & z\end{array}$.

Hence the insertion is consistent with the rule $u \rightarrow x z^{-1} y v$, which produces $j$ permutations on $[n+1]$ with $i+1$ exterior peaks of pattern 132, $j-1$ exterior peaks of pattern 231 and $k+1$ proper double descents. The total weight of these $j$ permutations is

$$
j x^{i+1} v^{i+1} u^{j-1} z^{j} y^{k+1} w^{n-2 i-2 j-k} .
$$

Case4. Insert $n+1$ before some element labeled by $z$. There exist two subcases. If $n+1$ is inserted before 0 , then we label $n+1$ by $w$ :

$$
\pi_{n} \begin{aligned}
& 0 \\
& z
\end{aligned} \Longrightarrow \begin{gathered}
\pi_{n}<\begin{array}{c}
n+1 \\
w
\end{array} \\
z
\end{gathered} .
$$

For example, inserting 7 before element 0 in (2.8), we get

$\begin{array}{llllllll}5 & 3 & 4 & 6 & 2 & 1 & 7 & 0 \\ x & v & w & u & z & y & w & z\end{array}$.

If $n+1$ is inserted before $\pi_{\ell}$ with label $z$ for $1 \leq \ell \leq n-1$, then the label of $n+1$ is assigned $u$, and the labele of $\pi_{\ell-1}$ is adjusted to $w$, as showed below:

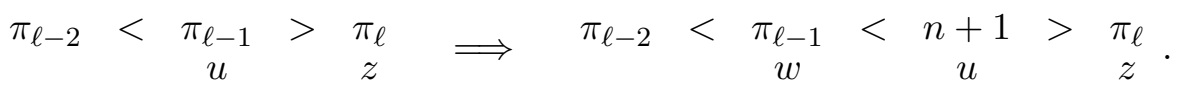

For example, inserting 7 before the element 2 in (2.8) yields

$$
\begin{array}{llllllll}
5 & 3 & 4 & 6 & 7 & 2 & 1 & 0 \\
x & v & w & w & u & z & y & z
\end{array} .
$$

In conclusion, the insertions in this case always correspond to the substitution rule $z \rightarrow z w$ and yields $j+1$ permutations on $[n+1]$ with $i$ exterior peaks of pattern $132, j$ exterior peaks of pattern 231, and $k$ proper double descents. Thus, we obtain the total weight

$$
(j+1) x^{i} v^{i} u^{j} z^{j+1} y^{k} w^{n-2 i-2 j-k+1} .
$$

Case 5. Insert $n+1$ before some element $\pi_{\ell}$ with label $w$. By the labeling rule, we know that $\pi_{\ell-1}<\pi_{\ell}$. Thus the position of $n+1$ becomes an exterior peak of pattern 132 then we label $n+1$ by $x$ and relabel $\pi_{\ell}$ by $v$ :

$$
\pi_{\ell-1}<\begin{gathered}
\pi_{\ell} \\
w
\end{gathered} \Longrightarrow \pi_{\ell-1}<\begin{array}{cc}
n+1 & > \\
x & \pi_{\ell} \\
v
\end{array} .
$$


For example, we insert 7 before the element 4 in (2.8), which changes the grammatical labeling to

$$
\begin{array}{llllllll}
5 & 3 & 7 & 4 & 6 & 2 & 1 & 0 \\
x & v & x & v & u & z & y & z
\end{array}
$$

This insertion corresponds to the rule $w \rightarrow x v$, which generates $n-2 i-2 j-k$ permutations on $[n+1]$ with $i+1$ exterior peaks of pattern 132, $j$ exterior peaks of pattern 231 and $k$ proper double descents. Thus the total weight of these permutations equals

$$
(n-2 i-2 j-k) x^{i+1} v^{i+1} u^{j} z^{j+1} y^{k} w^{n-2 i-2 j-k-1} .
$$

Case 6. Insert $n+1$ before some element $\pi_{\ell}$ labeled by $y$. It is easy to check that the position of $n+1$ becomes an exterior peak of pattern 231 since $\pi_{\ell-1}>\pi_{\ell}$. Thus we label $n+1$ by $u$ and relabel $\pi_{\ell}$ by $z$ :

$$
\pi_{\ell-2}>\pi_{\ell-1}>\pi_{\ell} \Longrightarrow \pi_{\ell-2}>\pi_{\ell-1}<\begin{gathered}
n+1>\pi_{\ell} \\
u
\end{gathered}
$$

For example, inserting 7 before the element 1 in (2.8) gives

$$
\begin{array}{llllllll}
5 & 3 & 4 & 6 & 2 & 7 & 1 & 0 \\
x & v & w & u & z & u & z & z
\end{array} .
$$

Hence, this insertion is coincident with the grammatical rule $y \rightarrow z u$ and produces $k$ permutations on $[n+1]$ with $i$ exterior peaks of pattern $132, j+1$ exterior peaks of pattern 231 and $k-1$ proper double descents. The total weight of these permutations amounts to

$$
k x^{i} v^{i} u^{j+1} z^{j+2} y^{k-1} w^{n-2 i-2 j-k} .
$$

Taking all the above six cases into account, we deduce that

$$
\begin{aligned}
& P_{n+1}(x, y, z, w, u, v)=\sum_{\pi \in \mathfrak{S}_{n+1}} w(\pi) \\
& =\sum_{i, j, k=0}^{n} P_{n}(i, j, k)\left(i x^{i} v^{i} u^{j} z^{j+1} y^{k+1} w^{n-2 i-2 j-k}+i x^{i-1} v^{i-1} u^{j+1} z^{j+2} y^{k+1} w^{n-2 i-2 j-k}\right. \\
& \quad+j x^{i+1} v^{i+1} u^{j-1} z^{j} y^{k+1} w^{n-2 i-2 j-k}+(j+1) x^{i} v^{i} u^{j} z^{j+1} y^{k} w^{n-2 i-2 j-k+1} \\
& \left.\quad+(n-2 i-2 j-k) x^{i+1} v^{i+1} u^{j} z^{j+1} y^{k} w^{n-2 i-2 j-k-1}+k x^{i} v^{i} u^{j+1} z^{j+2} y^{k-1} w^{n-2 i-2 j-k}\right) .
\end{aligned}
$$

By the grammar (1.5), we have that

$$
\begin{aligned}
& D\left(x^{i} v^{i} u^{j} z^{j+1} y^{k} w^{n-2 i-2 j-k}\right) \\
& =i x^{i} v^{i} u^{j} z^{j+1} y^{k+1} w^{n-2 i-2 j-k}+i x^{i-1} v^{i-1} u^{j+1} z^{j+2} y^{k+1} w^{n-2 i-2 j-k} \\
& \quad+j x^{i+1} v^{i+1} u^{j-1} z^{j} y^{k+1} w^{n-2 i-2 j-k}+(j+1) x^{i} v^{i} u^{j} z^{j+1} y^{k} w^{n-2 i-2 j-k+1} \\
& \quad+(n-2 i-2 j-k) x^{i+1} v^{i+1} u^{j} z^{j+1} y^{k} w^{n-2 i-2 j-k-1}+k x^{i} v^{i} u^{j+1} z^{j+2} y^{k-1} w^{n-2 i-2 j-k} .
\end{aligned}
$$


Therefore,

$$
P_{n+1}(x, y, z, w, u, v)=\sum_{\pi \in \mathfrak{S}_{n}} D(w(\pi)) .
$$

On the other hand, it follows from induction hypothesis that

$$
D^{n+1}(z)=D\left(D^{n}(z)\right)=D\left(\sum_{\pi \in \mathfrak{S}_{n}} w(\pi)\right)=\sum_{\pi \in \mathfrak{S}_{n}} D(w(\pi)) .
$$

Thus the theorem holds for $n+1$, which completes the proof.

By considering a different grammatical labeling on permutations, which is also consistent with the substitution rules of the grammar $G(1.5)$, we give a grammatical interpretation for the generating function $Q_{n}(x, y, z, w, u, v)$.

Theorem 2.2 Let $D$ be the formal derivative with respect to the grammar $G(1.5)$. For $n \geq 1$, we have

$$
D^{n}(w)=Q_{n}(x, y, z, w, u, v)
$$

Proof of Theorem 2.2. The proof is similar to that of Theorem 2.1, so we only present the corresponding grammatical labeling. Given a permutation $\pi=\pi_{1} \pi_{2} \cdots \pi_{n}$ on $[n]$, first add an element $\pi_{n+1}=0$ at the end of $\pi$. Then for $1 \leq i \leq n$, if $i$ is a peak of pattern 132, we label $\pi_{i}$ by $x$ and $\pi_{i+1}$ by $v$, if $i$ is a peak of pattern 231, we label $\pi_{i}$ by $u$ and $\pi_{i+1}$ by $z$, if $i$ is a double descent, we label $\pi_{i+1}$ by $y$, finally, if $i$ is a double rise, we label $\pi_{i}$ by $w$.

We claim that this grammatical labeling is well-defined, that is, for $1 \leq i \leq n+1$, each element $\pi_{i}$ is assigned exactly one label. Since $n=1$ is trivial, we assume $n \geq 2$. If $i=1$, then $\pi_{1}$ is either a peak of pattern 132 or a double rise since $0=\pi_{0}<\pi_{1}$. Thus $\pi_{1}$ is labeled by $x$ or $w$. If $i=n+1$, since $\pi_{n}$ is either a peak of pattern 231 or a double descent due to $\pi_{n}>\pi_{n+1}=0$, we may label $\pi_{n+1}$ by either $z$ or $y$. Suppose $2 \leq i \leq n$. If $\pi_{i-1}<\pi_{i}<\pi_{i+1}$, then $\pi_{i}$ is a double rise receiving label $w$, if $\pi_{i-1}<\pi_{i}>\pi_{i+1}$, then $\pi_{i}$ is a peak receiving label $x$ or label $u$. On the other hand, if $\pi_{i-2}>\pi_{i-1}>\pi_{i}$, then $\pi_{i-1}$ is a double descent so that we label $\pi_{i}$ by $y$, if $\pi_{i-2}<\pi_{i-1}>\pi_{i}$, then $\pi_{i-1}$ is a peak so that we label $\pi_{i}$ by either $z$ or $v$. Following the above labeling rules, we can always assign $\pi_{i}$ a label for $1 \leq i \leq n+1$, which completes the proof.

Furthermore, let $W_{n}(i, j, k, \ell, m)$ be the number of permutations on $[n]$ with $i$ peaks of pattern $132, j$ peaks of pattern $231, k$ double descents, $\ell$ double rises and $m-1$ valleys. Denote by

$$
W_{n}(x, y, z, w, u)=\sum_{i, j, k, \ell, m=0}^{n} W_{n}(i, j, k, \ell, m) x^{i} u^{j} z^{m} y^{k} w^{\ell}
$$

or equivalently,

$$
W_{n}(x, y, z, w, u)=\sum_{\pi \in \mathfrak{S}_{n}} x^{p_{1}(\pi)} u^{p_{2}(\pi)} z^{v(\pi)+1} y^{d d(\pi)} w^{d r(\pi)} .
$$

By the definitions of peaks and valleys, it is clear that the number of peaks in a permutation is one greater than that of valleys. If replacing $v$ by $z$ in $Q_{n}(x, y, z, w, u, v)$, then the exponent of $z$ will record the occurrences of valleys, that is,

$$
\left.Q_{n}(x, y, z, w, u, v)\right|_{v=z}=W_{n}(x, y, z, w, u) .
$$


Corollary 2.3 Let $D$ be the formal derivative with respect to the grammar $G$ (1.5). For $n \geq 1$, we have

$$
\left.D^{n}(w)\right|_{v=z}=W_{n}(x, y, z, w, u) .
$$

By using the Leibniz rule $(2.1)$ on $D^{n+1}(z)=D^{n}(z w)$, we have

$$
D^{n+1}(z)=\sum_{k=0}^{n}\left(\begin{array}{l}
n \\
k
\end{array}\right) D^{k}(z) D^{n-k}(w) .
$$

Hence for $n \geq 1$, combining Theorem 2.1 and Theorem 2.2, we can establish a convolutional relation:

$$
P_{n+1}(x, y, z, w, u, v)=\sum_{k=0}^{n}\left(\begin{array}{l}
n \\
k
\end{array}\right) P_{k}(x, y, z, w, u, v) Q_{n-k}(x, y, z, w, u, v) .
$$

\section{Connection to the parabolic cylinder functions}

In this section, by using the grammar

$$
G: x \rightarrow x y, y \rightarrow z u, z \rightarrow z w, w \rightarrow x v, u \rightarrow x y z^{-1} v, v \rightarrow x^{-1} z w u
$$

as in (1.5), we give the proofs of Theorem 1.5 and Theorem 1.9. To be more specific, combining Theorem 2.1 and (2.2), we have

$$
\sum_{n=0}^{\infty} P_{n}(x, y, z, w, u, v) \frac{t^{n}}{n !}=\operatorname{Gen}(z, t) .
$$

Similarly, by Theorem 2.2 and (2.2), along with the assumption that $Q_{0}(x, y, z, w, u, v)=w$, we find that

$$
\sum_{n=0}^{\infty} Q_{n}(x, y, z, w, u, v) \frac{t^{n}}{n !}=\operatorname{Gen}(w, t) .
$$

Therefore, to prove Theorem 1.5 and Theorem 1.9, we need to compute $\operatorname{Gen}(z, t)$ and $\operatorname{Gen}(w, t)$. To this end, we first give the explicit formula for the generating function $\operatorname{Gen}\left(x^{-1 / 2} z^{-1 / 2}, t\right)$ by solving the parabolic cylinder differential equation. Recall that the parabolic cylinder differential equation is a second-order ordinary differential equation of the form

$$
y^{\prime \prime}(z)+\left(a+\frac{1}{2}-\frac{z^{2}}{4}\right) y(z)=0
$$

whose solution can be written as

$$
y=c_{1} D_{a}(z)+c_{2} D_{-a-1}(i z),
$$

where $D_{a}(z)$ and $D_{-a-1}(i z)$ are independent, and $c_{1}, c_{2}$ are constants to be determined by the initial conditions of (3.3).

Based on the solutions of the equation (3.3), it is not difficult to solve a more general differential equation of the form

$$
y^{\prime \prime}(z)-\left(a z^{2}+b z+c\right) y(z)=0 .
$$


Theorem 3.1 For the general parabolic cylinder differential equation (3.4), all solutions can be written as

$$
c_{1} D_{\frac{b^{2}-4 a c-4 a^{3 / 2}}{8 a^{3 / 2}}}\left(\sqrt{2} a^{1 / 4} z+\frac{b}{\sqrt{2} a^{3 / 4}}\right)+c_{2} D_{\frac{4 a c-b^{2}-4 a^{3 / 2}}{8 a^{3 / 2}}}\left(i\left(\sqrt{2} a^{1 / 4} z+\frac{b}{\sqrt{2} a^{3 / 4}}\right)\right),
$$

where $c_{1}$ and $c_{2}$ are constants.

Proof. By equation (3.3), we know that these two parabolic cylinder functions are independent, thus we only need to prove that they are solutions of (3.4). To this end, we first introduce two recurrence relations related to $D_{a}(z)$ and $D_{a}^{\prime}(z)$ in $[19$, pp.16]:

$$
\begin{aligned}
& D_{a}^{\prime}(z)=\frac{1}{2} z D_{a}(z)-D_{a+1}(z) \\
& D_{a}^{\prime}(z)=a D_{a-1}(z)-\frac{1}{2} z D_{a}(z) .
\end{aligned}
$$

By (3.5) and (3.6), for any two constants $r$ and $s$, we can deduce that

$$
\begin{aligned}
D_{a}^{\prime}(r z+s) & =\frac{r}{2}(r z+s) D_{a}(r z+s)-r D_{a+1}(r z+s), \\
D_{a+1}^{\prime}(r z+s) & =r(a+1) D_{a}(r z+s)-\frac{r}{2}(r z+s) D_{a+1}(r z+s) .
\end{aligned}
$$

Combining (3.7) and (3.8) yields

$$
\begin{aligned}
D_{a}^{\prime \prime}(r z+s)= & \left.\frac{r}{2}(r z+s) D_{a}(r z+s)-r D_{a+1}(r z+s)\right)^{\prime} \\
= & \frac{r^{2}}{2} D_{a}(r z+s)+\frac{r}{2}(r z+s)\left(\frac{r}{2}(r z+s) D_{a}(r z+s)-r D_{a+1}(r z+s)\right) \\
& \quad-r^{2}\left((a+1) D_{a}(r z+s)-\frac{1}{2}(r z+s) D_{a+1}(r z+s)\right) \\
= & \frac{r^{2}}{4}\left((r z+s)^{2}-4 a-2\right) D_{a}(r z+s) .
\end{aligned}
$$

Therefore, plugging

$$
D_{\frac{b^{2}-4 a c-4 a^{3 / 2}}{8 a^{3 / 2}}}\left(\sqrt{2} a^{1 / 4} z+\frac{b}{\sqrt{2} a^{3 / 4}}\right) \text { and } D_{\frac{4 a c-b^{2}-4 a^{3 / 2}}{8 a^{3 / 2}}}\left(i\left(\sqrt{2} a^{1 / 4} z+\frac{b}{\sqrt{2} a^{3 / 4}}\right)\right)
$$

into (3.9), we see that both of them satisfy the general parabolic cylinder differential equation (3.4). This completes the proof.

The following theorem states that the generating function $\operatorname{Gen}\left(x^{-1 / 2} z^{-1 / 2}, t\right)$ satisfies a general parabolic cylinder differential equation.

Theorem 3.2 Let

$$
f(t)=\operatorname{Gen}\left(x^{-1 / 2} z^{-1 / 2}, t\right)
$$


and

$$
\alpha=(y+w)^{2}-2(x v+z u), \quad \beta=2(w-y)(x v-z u), \quad \gamma=2(x v-z u)^{2} .
$$

We have

$$
f^{\prime \prime}(t)-\left(\frac{\gamma}{8} t^{2}+\frac{\beta}{4} t+\frac{\alpha}{4}\right) f(t)=0
$$

Proof. By the grammar $G(1.5)$ and the corresponding formal derivative $D$, one can check that

$$
D^{2}\left(x^{-1 / 2} z^{-1 / 2}\right)=\frac{1}{4} x^{-1 / 2} z^{-1 / 2}\left((y+w)^{2}-2(x v+z u)\right)=\frac{\alpha}{4} x^{-1 / 2} z^{-1 / 2} .
$$

Moreover, since $D(x v-z u)=0$, it follows that

$$
D(\alpha)=\beta, \quad D(\beta)=\gamma, \quad D(\gamma)=4(x v-z u) D(x v-z u)=0 .
$$

By (2.4), we have

$$
f^{(4)}(t)=\sum_{n=0}^{\infty} D^{n+4}\left(x^{-1 / 2} z^{-1 / 2}\right) \frac{t^{n}}{n !} .
$$

From the Leibniz rule (2.1) and (3.11), we see that

$$
D^{n+4}\left(x^{-1 / 2} z^{-1 / 2}\right)=D^{n+2}\left(\frac{\alpha}{4} x^{-1 / 2} z^{-1 / 2}\right)=\frac{1}{4} \sum_{k=0}^{n+2}\left(\begin{array}{c}
n+2 \\
k
\end{array}\right) D^{k}(\alpha) D^{n+2-k}\left(x^{-1 / 2} z^{-1 / 2}\right) .
$$

By (3.12), the summation has only three nonzero terms, implying that

$$
\begin{aligned}
& D^{n+4}\left(x^{-1 / 2} z^{-1 / 2}\right) \\
& \quad=\frac{\alpha}{4} D^{n+2}\left(x^{-1 / 2} z^{-1 / 2}\right)+\frac{n+2}{4} \beta D^{n+1}\left(x^{-1 / 2} z^{-1 / 2}\right)+\frac{1}{4}\left(\begin{array}{c}
n+2 \\
2
\end{array}\right) \gamma D^{n}\left(x^{-1 / 2} z^{-1 / 2}\right) .
\end{aligned}
$$

Putting (3.14) into (3.13), and using (2.4) again, we deduce that

$$
f^{(4)}(t)-\left(\frac{\gamma}{8} t^{2}+\frac{\beta}{4} t+\frac{\alpha}{4}\right) f^{\prime \prime}(t)-\left(\frac{\gamma}{2} t+\frac{\beta}{2}\right) f^{\prime}(t)-\frac{\gamma}{4} f(t)=0 .
$$

Let

$$
g(t)=f^{\prime \prime}(t)-\left(\frac{\gamma}{8} t^{2}+\frac{\beta}{4} t+\frac{\alpha}{4}\right) f(t)
$$

Taking the second-order derivative with respect to $t$ on both sides gives

$$
g^{\prime \prime}(t)=f^{(4)}(t)-\left(\frac{\gamma}{8} t^{2}+\frac{\beta}{4} t+\frac{\alpha}{4}\right) f^{\prime \prime}(t)-\left(\frac{\gamma}{2} t+\frac{\beta}{2}\right) f^{\prime}(t)-\frac{\gamma}{4} f(t) .
$$

It follows from (3.15) that

$$
g^{\prime \prime}(t)=0 .
$$

Clearly, we have $g(t)=c_{1} t+c_{2}$ with the indeterminate constants $c_{1}$ and $c_{2}$, that is,

$$
f^{\prime \prime}(t)-\left(\frac{\gamma}{8} t^{2}+\frac{\beta}{4} t+\frac{\alpha}{4}\right) f(t)-c_{1} t-c_{2}=0 .
$$


Set $t=0$ and recall $f^{(n)}(0)=D^{n}\left(x^{-1 / 2} z^{-1 / 2}\right)$. By (3.11), we have

$$
c_{2}=f^{\prime \prime}(0)-\frac{\alpha}{4} f(0)=D^{2}\left(x^{-1 / 2} z^{-1 / 2}\right)-\frac{\alpha}{4} x^{-1 / 2} z^{-1 / 2}=0 .
$$

Hence, (3.16) can be reduced to

$$
f^{\prime \prime}(t)-\left(\frac{\gamma}{8} t^{2}+\frac{\beta}{4} t+\frac{\alpha}{4}\right) f(t)-c_{1} t=0
$$

Then taking the derivative with respect to $t$ on both sides yields

$$
f^{(3)}(t)-\left(\frac{\gamma}{8} t^{2}+\frac{\beta}{4} t+\frac{\alpha}{4}\right) f^{\prime}(t)-\left(\frac{\gamma}{4} t+\frac{\beta}{4}\right) f(t)-c_{1}=0 .
$$

Setting $t=0$ in $(3.17)$ gives

$$
\begin{aligned}
c_{1} & =f^{(3)}(0)-\frac{\alpha}{4} f^{\prime}(0)-\frac{\beta}{4} f(0) \\
& =D^{3}\left(x^{-1 / 2} z^{-1 / 2}\right)-\frac{\alpha}{4} D\left(x^{-1 / 2} z^{-1 / 2}\right)-\frac{\beta}{4} x^{-1 / 2} z^{-1 / 2} .
\end{aligned}
$$

Hence, from (3.11), we deduce that

$$
D^{3}\left(x^{-1 / 2} z^{-1 / 2}\right)=D\left(\frac{\alpha}{4} x^{-1 / 2} z^{-1 / 2}\right)=\frac{\alpha}{4} D\left(x^{-1 / 2} z^{-1 / 2}\right)+\frac{\beta}{4} x^{-1 / 2} z^{-1 / 2} .
$$

Combining (3.18) and (3.19), we see that $c_{1}=0$. Therefore, given $c_{1}=c_{2}=0$, equation (3.16) reduces to (3.10). This completes the proof.

Combing Theorem 3.1 and Theorem 3.2, we give the explicit form for the generating function $\operatorname{Gen}\left(x^{-1 / 2} z^{-1 / 2}, t\right)$ in terms of the parabolic cylinder functions.

Theorem 3.3 Let $\delta, \hat{\delta}, p, q, r, s$ be defined as in Theorem 1.5. We have

$$
\begin{gathered}
\operatorname{Gen}\left(x^{-1 / 2} z^{-1 / 2}, t\right) \\
=\frac{x^{-1 / 2} z^{-1 / 2}}{p q(w-y)+(\hat{\delta} p s-\delta q r)}\left((\hat{\delta} s-q y) D_{\frac{z u-y w}{\delta^{2}}}\left(\delta t+\frac{w-y}{\delta}\right)\right. \\
\left.+(p w-\delta r) D_{\frac{x v-y w}{\delta^{2}}}\left(\hat{\delta} t+\frac{y-w}{\hat{\delta}}\right)\right) .
\end{gathered}
$$

Proof. From Theorem 3.1 and Theorem 3.2, $\operatorname{Gen}\left(x^{-1 / 2} z^{-1 / 2}, t\right)$ can be expressed as follows

$$
\begin{aligned}
& \operatorname{Gen}\left(x^{-1 / 2} z^{-1 / 2}, t\right) \\
& \quad=c_{1} D_{\frac{z u-y w}{x v-z u}}\left(\sqrt{x v-z u} t+\frac{w-y}{\sqrt{x v-z u}}\right)+c_{2} D_{\frac{y w-x v}{x v-z u}}\left(i\left(\sqrt{x v-z u} t+\frac{w-y}{\sqrt{x v-z u}}\right)\right)
\end{aligned}
$$


with the indeterminate constants $c_{1}$ and $c_{2}$. By noticing that $i \sqrt{x v-z u}=\sqrt{z u-x v}$, we rewrite $\operatorname{Gen}\left(x^{-1 / 2} z^{-1 / 2}, t\right)$ as

$$
\begin{aligned}
& \operatorname{Gen}\left(x^{-1 / 2} z^{-1 / 2}, t\right) \\
& \quad=c_{1} D_{\frac{z u-y w}{x v-z u}}\left(\sqrt{x v-z u} t+\frac{w-y}{\sqrt{x v-z u}}\right)+c_{2} D_{\frac{x v-y w}{z u-x v}}\left(\sqrt{z u-x v} t+\frac{y-w}{\sqrt{z u-x v}}\right) .
\end{aligned}
$$

Let $\delta, \hat{\delta}, p, q, r, s$ be defined as in Theorem 1.5. Since $\operatorname{Gen}\left(x^{-1 / 2} z^{-1 / 2}, 0\right)=x^{-1 / 2} z^{-1 / 2}$, we obtain

$$
c_{1} p+c_{2} q=x^{-1 / 2} z^{-1 / 2}
$$

Moreover,

$$
\operatorname{Gen}^{\prime}\left(x^{-1 / 2} z^{-1 / 2}, 0\right)=D\left(x^{-1 / 2} z^{-1 / 2}\right)=-\frac{1}{2} x^{-1 / 2} z^{-1 / 2}(y+w) .
$$

Thus, by (3.7), we see that

$$
c_{1}\left(\frac{w-y}{2} p-\delta r\right)+c_{2}\left(\frac{y-w}{2} q-\hat{\delta} s\right)=-\frac{1}{2} x^{-1 / 2} z^{-1 / 2}(y+w) .
$$

Combining (3.21) and (3.22), we arrive at

$$
c_{1}=\frac{(\hat{\delta} s-q y) x^{-1 / 2} z^{-1 / 2}}{p q(w-y)+(\hat{\delta} p s-\delta q r)} \quad \text { and } \quad c_{2}=\frac{(p w-\delta r) x^{-1 / 2} z^{-1 / 2}}{p q(w-y)+(\hat{\delta} p s-\delta q r)} .
$$

This completes the proof.

Now we are ready to prove Theorem 1.5 and Theorem 1.9.

Proof of Theorem 1.5. Using mathematical induction on $n$ with the fact $D^{2}(w-y)=D(x v-$ $z u)=0$, we see that for $n \geq 0$,

$$
D^{n}\left(x^{-1} z\right)=x^{-1} z \sum_{k=0}^{\lfloor n / 2\rfloor} \frac{n !}{2^{k}(n-2 k) ! k !}(w-y)^{n-2 k}(x v-z u)^{k} .
$$

Therefore, we have

$$
\begin{aligned}
\operatorname{Gen}\left(x^{-1} z, t\right) & =\sum_{n=0}^{\infty} x^{-1} z\left(\sum_{k=0}^{\lfloor n / 2\rfloor} \frac{n !}{2^{k}(n-2 k) ! k !}(w-y)^{n-2 k}(x v-z u)^{k}\right) \frac{t^{n}}{n !} \\
& =x^{-1} z \sum_{k=0}^{\infty} \sum_{n=0}^{\infty} \frac{(w-y)^{n}(x v-z u)^{k} t^{n+2 k}}{2^{k} n ! k !} \\
& =x^{-1} z e^{(w-y) t+\frac{(x v-z u) t^{2}}{2}} .
\end{aligned}
$$

On the other hand, by (2.3), we have

$$
\left(\operatorname{Gen}(z, t) \cdot \operatorname{Gen}\left(x^{-1 / 2} z^{-1 / 2}, t\right)\right)^{2}=\operatorname{Gen}\left(x^{-1 / 2} z^{1 / 2}, t\right)^{2}=\operatorname{Gen}\left(x^{-1} z, t\right)
$$


which leads to

$$
\operatorname{Gen}(z, t)=\frac{\operatorname{Gen}\left(x^{-1} z, t\right)^{1 / 2}}{\operatorname{Gen}\left(x^{-1 / 2} z^{-1 / 2}, t\right)}
$$

Plugging (3.20) and (3.23) into (3.24), we arrive at the generating function $\operatorname{Gen}(z, t)$.

Proof of Theorem 1.9. From the substitution rule $z \rightarrow z w$ in the grammar (1.5), utilizing (2.3) and (2.4), we obtain that

$$
\operatorname{Gen}^{\prime}(z, t)=\operatorname{Gen}(D(z), t)=\operatorname{Gen}(z w, t)=\operatorname{Gen}(z, t) \operatorname{Gen}(w, t),
$$

thus,

$$
\operatorname{Gen}(w, t)=\frac{\operatorname{Gen}^{\prime}(z, t)}{\operatorname{Gen}(z, t)} .
$$

Using (3.7) on Gen $(z, t)$ to compute (3.25), we are led to Theorem 1.9.

To see that Theorem 1.5 implies Theorem 1.4, replacing $u$ by $x$ and $v$ by $z$ in (1.4) gives (1.2), that is,

$$
\left.P_{n}(x, y, z, w, u, v)\right|_{\substack{u=x \\ v=z}}=P_{n}(x, y, z, w) .
$$

Thus by (3.1), we derive

$$
\sum_{n=0}^{\infty} P_{n}(x, y, z, w) \frac{t^{n}}{n !}=\left.\operatorname{Gen}(z, t)\right|_{\substack{u=x \\ v=z}}
$$

It follows from (3.24) that

$$
\left.\operatorname{Gen}(z, t)\right|_{\substack{u=x \\ v=z}}=\frac{\left(\left.\operatorname{Gen}\left(x^{-1} z, t\right)\right|_{\substack{u=x \\ v=z}}\right)^{1 / 2}}{\left.\operatorname{Gen}\left(x^{-1 / 2} z^{-1 / 2}, t\right)\right|_{\substack{u=x \\ v=z}}} .
$$

From (3.23), we can directly deduce that

$$
\left.\operatorname{Gen}\left(x^{-1} z, t\right)\right|_{\substack{u=x \\ v=z}}=x^{-1} z e^{(w-y) t} .
$$

With the replacement $u \rightarrow x$ and $v \rightarrow z$, the parabolic cylinder differential equation (3.10) reduces to the second order linear differential equation

$$
h^{\prime \prime}(t)-((y+w)-4 x z) h(t)=0,
$$

where $h(t)=\left.\operatorname{Gen}\left(x^{-1 / 2} z^{-1 / 2}, t\right)\right|_{\substack{u=x \\ v=z}}$. According to the initial conditions

$$
h(0)=x^{-\frac{1}{2}} z^{-\frac{1}{2}} \quad \text { and } \quad h^{\prime}(0)=-\frac{1}{2} x^{-\frac{1}{2}} z^{-\frac{1}{2}}(y+w),
$$

solving the linear differential equation gives

$$
\begin{aligned}
& \left.\operatorname{Gen}\left(x^{-\frac{1}{2}} z^{-\frac{1}{2}}, t\right)\right|_{\substack{u=x \\
v=z}} \\
& =\frac{x^{-\frac{1}{2}} z^{-\frac{1}{2}}}{2}\left(\left(1+\frac{y+w}{\sqrt{(y+w)^{2}-4 x z}}\right) e^{-\frac{t}{2} \sqrt{(y+w)^{2}-4 x z}}\right.
\end{aligned}
$$




$$
\left.+\left(1-\frac{y+w}{\sqrt{(y+w)^{2}-4 x z}}\right) e^{\frac{t}{2} \sqrt{(y+w)^{2}-4 x z}}\right) .
$$

Hence, plugging (3.27) and (3.28) into (3.26), we arrive at

$$
\begin{aligned}
& \left.\operatorname{Gen}(z, t)\right|_{\substack{u=x \\
v=z}} \\
& =\frac{2 z \sqrt{(y+w)^{2}-4 x z} e^{\frac{t}{2}\left(w-y+\sqrt{(y+w)^{2}-4 x z}\right)}}{y+w+\sqrt{(y+w)^{2}-4 x z}-\left(y+w-\sqrt{(y+w)^{2}-4 x z}\right) e^{t \sqrt{(y+w)^{2}-4 x z}}},
\end{aligned}
$$

which proves Theorem 1.4.

Next we show that Theorem 1.9 implies Theorem 1.8. Substituting $u$ by $x$ in (2.9) and comparing with (1.9), then for $n \geq 1$, we deduce

$$
\left.W_{n}(x, y, z, w, u)\right|_{u=x}=x z F_{n}(x, y, z, w) .
$$

Together with (2.10), we have

$$
\left.Q_{n}(x, y, z, w, u)\right|_{\substack{u=x \\ v=z}} ^{u}=x z F_{n}(x, y, z, w) .
$$

By (3.2), we see

$$
\begin{aligned}
\sum_{n=1}^{\infty} F_{n}(x, y, z, w) \frac{t^{n}}{n !} & =\left.x^{-1} z^{-1} \sum_{n=1}^{\infty} Q_{n}(x, y, z, w, u, v)\right|_{\substack{u=x \\
v=z}} \frac{t^{n}}{n !} \\
& =x^{-1} z^{-1}\left(\left.\operatorname{Gen}(w, t)\right|_{\substack{u=x \\
v=z}}-w\right) .
\end{aligned}
$$

It follow from (3.25) that

$$
\left.\operatorname{Gen}(w, t)\right|_{\substack{u=x \\ v=z}}=\frac{\left(\left.\operatorname{Gen}(z, t)\right|_{\substack{u=x \\ v=z}}\right)^{\prime}}{\left.\operatorname{Gen}(z, t)\right|_{\substack{u=x \\ v=z}}}
$$

Let $\alpha \beta=x z$ and $\alpha+\beta=y+w$, then by (3.29) and (3.31), we derive

$$
\left.\operatorname{Gen}(w, t)\right|_{\substack{u=x \\ v=z}}=\alpha \beta \frac{e^{\beta t}-e^{\alpha t}}{\beta e^{\alpha t}-\alpha e^{\beta t}}+w .
$$

Therefore, plugging (3.32) into (3.30) proves Theorem 1.8.

\section{Specializations}

In this section, we discuss several applications of Theorem 1.5 and Theorem 1.9 by specializing the variables in $\{x, y, z, w, u, v\}$. Before this, we recall the definition of consecutive patterns [9] in permutations. Let $m \leq n$ be two positive integers, and $\pi=\pi_{1} \pi_{2} \cdots \pi_{n}$ be a permutation on $[n]$ and $\sigma=\sigma_{1} \sigma_{2} \cdots \sigma_{m}$ be a permutation on $[m]$. Then we say $\pi$ contains a consecutive pattern $\sigma$ if there exist $m$ consecutive elements $\pi_{i+1} \pi_{i+2} \cdots \pi_{i+m}$ in $\pi(0 \leq i \leq n-m)$ such 
that $\rho\left(\pi_{i+1} \pi_{i+2} \cdots \pi_{i+m}\right)=\sigma$, where $\rho$ is the reduction consisting in relabeling the elements with $\{1,2, \ldots, m\}$ so that they keep the same order relationships they have in $\pi$.

Let $L(n, k)$ be the number of permutations of $[n]$ with $k$ consecutive patterns 231 and 321 , and let

$$
L_{n}(x)=\sum_{k \geq 0} L(n, k) x^{k}
$$

In other words, $L(n, k)$ counts the number of permutations of $[n]$ whose total number of exterior peaks of pattern 231 and proper double descents is $k$.

Proof of Theorem 1.6. Note that by (1.3), we have

$$
P_{n}(1, x, 1,1, x, 1)=L_{n}(x) .
$$

Due to the explicit form of $D_{a}(z)$ in $(1.6)$ with the fact $\lim _{z \rightarrow 0} \frac{1}{\Gamma(z)}=0$, we can easily obtain

$$
D_{0}(z)=e^{-\frac{z^{2}}{4}} \text { and } D_{1}(z)=z e^{-\frac{z^{2}}{4}} \text {. }
$$

Moreover, we see that

$$
D_{-1}(z)=\sqrt{\frac{\pi}{2}} e^{-\frac{z^{2}}{4}}\left(e^{\frac{z^{2}}{2}}-\frac{\sqrt{2} z}{\sqrt{\pi}}{ }_{1} F_{1}\left(1 ; \frac{3}{2} ; \frac{z^{2}}{2}\right)\right)=\sqrt{\frac{\pi}{2}} e^{\frac{z^{2}}{4}}-z e^{-\frac{z^{2}}{4}}{ }_{1} F_{1}\left(1 ; \frac{3}{2} ; \frac{z^{2}}{2}\right) .
$$

Notice that

$$
{ }_{1} F_{1}\left(1 ; \frac{3}{2} ; z^{2}\right)=\frac{\sqrt{\pi}}{2 z} e^{z^{2}} \operatorname{erf}(z),
$$

where $\operatorname{erf}(x)$ is the error function defined by

$$
\operatorname{erf}(x)=\frac{2}{\sqrt{\pi}} \int_{0}^{x} e^{-s^{2}} d s .
$$

Then setting $z \rightarrow \frac{z}{\sqrt{2}}$ in (4.4) gives

$$
{ }_{1} F_{1}\left(1 ; \frac{3}{2} ; \frac{z^{2}}{2}\right)=\sqrt{\frac{\pi}{2}} z^{-1} e^{\frac{z^{2}}{2}} \operatorname{erf}\left(\frac{z}{\sqrt{2}}\right)
$$

which leads to

$$
D_{-1}(z)=\sqrt{\frac{\pi}{2}} e^{\frac{z^{2}}{4}}\left(1-\operatorname{erf}\left(\frac{z}{\sqrt{2}}\right)\right) .
$$

Therefore, by (4.2), setting $x, z, w, v \rightarrow 1$ and $y, u \rightarrow x$ in Theorem 1.5, utilizing (4.3) and (4.5), we have

$$
\sum_{n=0}^{\infty} \frac{L_{n}(x)}{n !} t^{n}=\frac{\sqrt{2(x-1)} e^{\frac{t(t+2)(1-x)}{2}}}{\sqrt{2(x-1)}+\sqrt{\pi} e^{\frac{x-1}{2}} x\left(\operatorname{erf}\left(\sqrt{\frac{x-1}{2}}\right)-\operatorname{erf}\left(\frac{(t+1) \sqrt{x-1}}{\sqrt{2}}\right)\right)} .
$$

After replacing the error function $\operatorname{erf}(x)$ by integration, we finally arrive at (1.7). This completes the proof. 
By setting $x=0$ in (4.1), we have $L_{n}(0)=L(n, 0)$, which is the number of permutations on $[n]$ avoiding the consecutive patterns 231 and 321 . It follows from Theorem 1.6 that

$$
\sum_{n=0}^{\infty} \frac{L_{n}(0)}{n !} t^{n}=e^{t+\frac{t^{2}}{2}}
$$

Note that (4.6) is also the generating function of the number of involutions on $[n]$, see [15, Eq. $5.32]$, which implies that the number of permutations on $[n]$ avoiding the consecutive patterns 231 and 321 equals the number of involutions on $[n]$. A bijective proof given by Callan can be found in [20, A000085].

Let $T(n, i, j)$ be the number of permutations on $[n]$ with $i$ exterior peaks of pattern 132 and $j$ exterior peaks of pattern 231, and let

$$
T_{n}(x, y)=\sum_{i, j \geq 0} T(n, i, j) x^{i} y^{j} .
$$

Proof of Theorem 1.7. By (1.3), we know that $P_{n}(x, 1,1,1, y, 1)=T_{n}(x, y)$. Thus after replacements $y, z, w, v \rightarrow 1$ and $u \rightarrow y$ in Theorem 1.5, we obtain the numerator as

$$
\lim _{\substack{y, z, w, v \rightarrow 1 \\ u \rightarrow y}} z(p q(w-y)+(\hat{\delta} p s-\delta q r)) e^{\frac{w-y}{2} t+\frac{\delta^{2}}{4} t^{2}}=\pi e^{\frac{x-y}{4} t^{2}} \Theta
$$

where

$$
\Theta=\frac{\sqrt{y-x}}{\Gamma\left(\frac{1-x}{2(y-x)}\right) \Gamma\left(\frac{1}{2}+\frac{1-y}{2(x-y)}\right)}-\frac{\sqrt{x-y}}{\Gamma\left(\frac{1-y}{2(x-y)}\right) \Gamma\left(\frac{1}{2}+\frac{1-x}{2(y-x)}\right)} .
$$

For the denominator, we have

$$
\begin{aligned}
\lim _{\substack{y, z, w, v \rightarrow 1 \\
u \rightarrow y}}(\hat{\delta} s-q y) D_{\frac{z u-y w}{\delta^{2}}}\left(\delta t+\frac{w-y}{\delta}\right) \\
=-\frac{\pi e^{\frac{y-x}{4} t^{2}}{ }_{1} F_{1}\left(\frac{1-y}{2(x-y)} ; \frac{1}{2} ; \frac{x-y}{2} t^{2}\right)}{\sqrt{2} \Gamma\left(\frac{1}{2}+\frac{1-x}{2(y-x)}\right) \Gamma\left(\frac{1}{2}+\frac{1-y}{2(x-y)}\right)}-\frac{\pi e^{\frac{y-x}{4} t^{2}} t \sqrt{-2(x-y)^{2}}{ }_{1} F_{1}\left(\frac{1}{2}+\frac{1-y}{2(x-y)} ; \frac{3}{2} ; \frac{x-y}{2} t^{2}\right)}{\Gamma\left(\frac{1-x}{2(y-x)}\right) \Gamma\left(\frac{1-y}{2(x-y)}\right)} \\
+\frac{\pi e^{\frac{y-x}{4} t^{2}} \sqrt{y-x_{1}} F_{1}\left(\frac{1-y}{2(x-y)} ; \frac{1}{2} ; \frac{x-y}{2} t^{2}\right)}{\Gamma\left(\frac{1-x}{2(y-x)}\right) \Gamma\left(\frac{1}{2}+\frac{1-y}{2(x-y)}\right)}+\frac{\pi e^{\frac{y-x}{4} t^{2}} t \sqrt{x-y} F_{1}\left(\frac{1}{2}+\frac{1-y}{2(x-y)} ; \frac{3}{2} ; \frac{x-y}{2} t^{2}\right)}{\Gamma\left(\frac{1-y}{2(x-y)}\right) \Gamma\left(\frac{1}{2}+\frac{1-x}{2(y-x)}\right)} \\
=-A_{1}-A_{2}+A_{3}+A_{4},
\end{aligned}
$$

and

$$
\begin{aligned}
& \lim _{\substack{y, z, w, v \rightarrow 1 \\
u \rightarrow y}}(p w-\delta r) D_{\frac{x v-y w}{\hat{\delta}^{2}}}\left(\hat{\delta} t+\frac{y-w}{\hat{\delta}}\right) \\
& \quad=\frac{\pi e^{\frac{x-y}{4} t^{2}}{ }_{1} F_{1}\left(\frac{1-x}{2(y-x)} ; \frac{1}{2} ; \frac{y-x}{2} t^{2}\right)}{\sqrt{2} \Gamma\left(\frac{1}{2}+\frac{1-x}{2(y-x)}\right) \Gamma\left(\frac{1}{2}+\frac{1-y}{2(x-y)}\right)}+\frac{\pi e^{\frac{x-y}{4} t^{2}} t \sqrt{-2(x-y)^{2}}{ }_{1} F_{1}\left(\frac{1}{2}+\frac{1-x}{2(y-x)} ; \frac{3}{2} ; \frac{y-x}{2} t^{2}\right)}{\Gamma\left(\frac{1-x}{2(y-x)}\right) \Gamma\left(\frac{1-y}{2(x-y)}\right)}
\end{aligned}
$$




$$
\begin{aligned}
& -\frac{\pi e^{\frac{x-y}{4} t^{2}} \sqrt{x-y} F_{1}\left(\frac{1-x}{2(y-x)} ; \frac{1}{2} ; \frac{y-x}{2} t^{2}\right)}{\Gamma\left(\frac{1-y}{2(x-y)}\right) \Gamma\left(\frac{1}{2}+\frac{1-x}{2(y-x)}\right)}-\frac{\pi e^{\frac{x-y}{4} t^{2}} t \sqrt{y-x_{1}} F_{1}\left(\frac{1}{2}+\frac{1-x}{2(y-x)} ; \frac{3}{2} ; \frac{y-x}{2} t^{2}\right)}{\Gamma\left(\frac{1-x}{2(y-x)}\right) \Gamma\left(\frac{1}{2}+\frac{1-y}{2(x-y)}\right)} \\
= & B_{1}+B_{2}-B_{3}-B_{4} .
\end{aligned}
$$

Since

$$
{ }_{1} F_{1}(a ; b ; z)=e^{z}{ }_{1} F_{1}(b-a ; b ;-z)
$$

we deduce that

$$
\begin{gathered}
{ }_{1} F_{1}\left(\frac{1-x}{2(y-x)} ; \frac{1}{2} ; \frac{y-x}{2} t^{2}\right)=e^{\frac{y-x}{2} t^{2}}{ }_{1} F_{1}\left(\frac{1-y}{2(x-y)} ; \frac{1}{2} ; \frac{x-y}{2} t^{2}\right), \\
{ }_{1} F_{1}\left(\frac{1}{2}+\frac{1-x}{2(y-x)} ; \frac{3}{2} ; \frac{y-x}{2} t^{2}\right)=e^{\frac{y-x}{2} t^{2}}{ }_{1} F_{1}\left(\frac{1}{2}+\frac{1-y}{2(x-y)} ; \frac{3}{2} ; \frac{x-y}{2} t^{2}\right) .
\end{gathered}
$$

Plugging (4.10) into $B_{1}$ and $B_{3}$, and plugging (4.11) into $B_{2}$ and $B_{4}$, we see that $A_{1}=B_{1}$, $A_{2}=B_{2}$ and

$$
\begin{aligned}
& A_{3}-B_{3}=\pi e^{\frac{y-x}{4} t^{2}} \Theta_{1} F_{1}\left(\frac{1-y}{2(x-y)} ; \frac{1}{2} ; \frac{x-y}{2} t^{2}\right) \\
& A_{4}-B_{4}=-\pi e^{\frac{y-x}{4} t^{2}} \Theta t_{1} F_{1}\left(\frac{1}{2}+\frac{1-y}{2(x-y)} ; \frac{3}{2} ; \frac{x-y}{2} t^{2}\right),
\end{aligned}
$$

which implies

$$
\begin{aligned}
\lim _{\substack{y, z, w, v \rightarrow 1 \\
u \rightarrow y}}(\hat{\delta} s-q y) D_{\frac{z u-y w}{\delta^{2}}}\left(\delta t+\frac{w-y}{\delta}\right)+(p w-\delta r) D_{\frac{x v-y w}{\delta^{2}}}\left(\hat{\delta} t+\frac{y-w}{\hat{\delta}}\right) \\
=\pi e^{\frac{y-x}{4} t^{2}} \Theta\left({ }_{1} F_{1}\left(\frac{1-y}{2(x-y)} ; \frac{1}{2} ; \frac{x-y}{2} t^{2}\right)-t_{1} F_{1}\left(\frac{1}{2}+\frac{1-y}{2(x-y)} ; \frac{3}{2} ; \frac{x-y}{2} t^{2}\right)\right) .
\end{aligned}
$$

Therefore, combining (4.8) and (4.12) gives (1.8), which completes the proof.

Letting $y \rightarrow x$ in Theorem 1.7 also leads to the result of Gessel [20, A008971].

Proof of Theorem 1.1. One can check that

$$
\lim _{y \rightarrow x} F_{1}\left(\frac{1-y}{2(x-y)} ; \frac{1}{2} ; \frac{x-y}{2} t^{2}\right)=\sum_{n=0}^{\infty} \frac{(\sqrt{1-x} t)^{2 n}}{(2 n) !}=\cosh (\sqrt{1-x} t)
$$

and

$$
\lim _{y \rightarrow x} F_{1} F_{1}\left(\frac{1}{2}+\frac{1-y}{2(x-y)} ; \frac{3}{2} ; \frac{x-y}{2} t^{2}\right)=\frac{1}{\sqrt{1-x} t} \sum_{n=0}^{\infty} \frac{(\sqrt{1-x} t)^{2 n+1}}{(2 n+1) !}=\frac{1}{\sqrt{1-x} t} \sinh (\sqrt{1-x} t) .
$$

Thus we have

$$
\lim _{y \rightarrow x} \sum_{n=0}^{\infty} T_{n}(x, y) \frac{t^{n}}{n !}=\lim _{y \rightarrow x} \frac{e^{\frac{x-y}{2} t^{2}}}{{ }_{1} F_{1}\left(\frac{1-y}{2(x-y)} ; \frac{1}{2} ; \frac{x-y}{2} t^{2}\right)-t_{1} F_{1}\left(\frac{1}{2}+\frac{1-y}{2(x-y)} ; \frac{3}{2} ; \frac{x-y}{2} t^{2}\right)}
$$




$$
\begin{aligned}
& =\frac{\sqrt{1-x}}{\sqrt{1-x} \cosh (\sqrt{1-x} t)-\sinh (\sqrt{1-x} t)} \\
& =\sum_{n=0}^{\infty} T_{n}(x) \frac{t^{n}}{n !} .
\end{aligned}
$$

We complete the proof.

Furthermore, setting $y \rightarrow 1$ and $x \rightarrow 1$ in (4.7), respectively, we deduce that

$$
\bar{T}_{n}(x):=T_{n}(x, 1)=\sum_{k \geq 0} T_{1}(n, k) x^{k} \quad \text { and } \quad \tilde{T}_{n}(y):=T_{n}(1, y)=\sum_{k \geq 0} T_{2}(n, k) y^{k}
$$

where $T_{1}(n, k)$ is the number of permutations on $[n]$ with $k$ exterior peaks of pattern 132 , and $T_{2}(n, k)$ is the number of permutations on $[n]$ with $k$ exterior peaks of pattern 231 .

Corollary 4.1 We have

$$
\sum_{n=0}^{\infty} \bar{T}_{n}(x) \frac{t^{n}}{n !}=\frac{e^{\frac{x-1}{2} t^{2}}}{1-\int_{0}^{t} e^{\frac{x-1}{2} s^{2}} d s}
$$

and

$$
\sum_{n=0}^{\infty} \tilde{T}_{n}(y) \frac{t^{n}}{n !}=\frac{1}{1-\int_{0}^{t} e^{\frac{y-1}{2} s^{2}} d s}
$$

Proof. Since

$$
{ }_{1} F_{1}\left(\frac{1}{2} ; \frac{3}{2} ;-z^{2}\right)=\frac{\sqrt{\pi}}{2 z} \operatorname{erf}(z)
$$

we observe that

$$
\lim _{y \rightarrow 1} F_{1}\left(\frac{1}{2}+\frac{1-y}{2(x-y)} ; \frac{3}{2} ; \frac{x-y}{2} t^{2}\right)=\frac{\sqrt{\pi}}{\sqrt{2(1-x)} t} \operatorname{erf}\left(\sqrt{\frac{1-x}{2}} t\right) .
$$

Then by Theorem 1.7, setting $y \rightarrow 1$ in (1.8) and utilizing (4.15), we obtain

$$
\sum_{n=0}^{\infty} \bar{T}_{n}(x) \frac{t^{n}}{n !}=\frac{\sqrt{2(1-x)} e^{\frac{x-1}{2} t^{2}}}{\sqrt{2(1-x)}-\sqrt{\pi} \operatorname{erf}\left(\sqrt{\frac{1-x}{2}} t\right)} .
$$

With the help of (4.4), we deduce that

$$
\lim _{x \rightarrow 1}{ }_{1} F_{1}\left(\frac{1}{2}+\frac{1-y}{2(x-y)} ; \frac{3}{2} ; \frac{x-y}{2} t^{2}\right)=\frac{\sqrt{\pi}}{\sqrt{2(1-y) t}} e^{\frac{1-y}{2} t^{2}} \operatorname{erf}\left(\sqrt{\frac{1-y}{2}} t\right) .
$$

Thus, setting $x \rightarrow 1$ in (1.8) and using (4.17), we see that

$$
\sum_{n=0}^{\infty} \tilde{T}_{n}(y) \frac{t^{n}}{n !}=\frac{\sqrt{2(1-y)}}{\sqrt{2(1-y)}-\sqrt{\pi} \operatorname{erf}\left(\sqrt{\frac{1-y}{2}} t\right)} .
$$


After replacement error functions by integration in (4.16) and (4.18), we obtain (4.13) and (4.14). This completes the proof.

Setting $x=0$ in (4.13), we obtain the generating function of the number of permutations on $[n]$ with no exterior peaks of pattern 132

$$
\sum_{n=0}^{\infty} T_{1}(n, 0) \frac{t^{n}}{n !}=\frac{e^{-\frac{t^{2}}{2}}}{1-\int_{0}^{t} e^{-\frac{s^{2}}{2}} d s},
$$

which recasts the formula obtained by Kiteav in [12, Theorem 6]. Since $\pi_{0} \pi_{1} \pi_{2}$ cannot be a exterior peak of pattern $231, T_{2}(n, k)$ is also the number of permutations on $[n]$ with $k$ consecutive pattern 231. Thus, (4.14) reproduces the result of Elizalde-Noy given in [9, Theorem 4.1]. Similarly, setting $y=0$ in (4.14) yields the generating functions of permutations on $[n]$ avoiding the consecutive patterns 231 :

$$
\sum_{n=0}^{\infty} T_{2}(n, 0) \frac{t^{n}}{n !}=\frac{1}{1-\int_{0}^{t} e^{-\frac{s^{2}}{2}} d s},
$$

which is also a special case of [9, Theorem 4.1] and [13, Theorem 12].

As the conclusion of this section, by combining Theorem 1.5 and Theorem 1.9, we give the joint distribution of exterior peaks of pattern 132 and of pattern 231 over alternating permutations on $[n]$. As defined in [15], we say that a permutation $\pi=\pi_{1} \pi_{2} \cdots \pi_{n} \in \mathfrak{S}_{n}$ is alternating (or zigzag or down-up) if $\pi_{1}>\pi_{2}<\pi_{3}>\pi_{4}<\cdots$. Recall that $T^{A}(n, i, j)$ is the number of alternating permutations on $[n]$ with $i$ exterior peaks of pattern 132 and $j$ exterior peaks of pattern 231, and the generating function

$$
T_{n}^{A}(x, y)=\sum_{i, j \geq 0} T^{A}(n, i, j) x^{i} y^{j}
$$

From (1.11), we see that

$$
\lim _{x, y \rightarrow 1}{ }_{1} F_{1}\left(\frac{x}{2(x-y)} ; \frac{3}{2} ;-\frac{x-y}{2} t^{2}\right)=\sum_{n=0}^{\infty} \frac{(-1)^{n}}{(2 n+1) !} t^{2 n}=\frac{\sin t}{t}
$$

and

$$
\lim _{x, y \rightarrow 1}{ }_{1} F_{1}\left(-\frac{y}{2(x-y)} ; \frac{1}{2} ; \frac{x-y}{2} t^{2}\right)=\sum_{n=0}^{\infty} \frac{(-1)^{n}}{(2 n) !} t^{2 n}=\cos t .
$$

Thus if we set $x, y \rightarrow 1$ in (1.11), then (1.11) reduces to the generating function

$$
\sum_{n=0}^{\infty} E_{n} \frac{t^{n}}{n !}=\sec t+\tan t
$$

where the Euler number $E_{n}$ is the number of alternating permutations on $[n]$.

Proof of Theorem 1.10. The proof is divided into the following cases depending on the parity of $n$. For the case that $n$ is even, we let $z, v \rightarrow 1$ and $y, w \rightarrow 0$ and $u \rightarrow y$ in (1.3). One can 
check that the permutations not vanished are alternating permutations with even length. Thus we have

$$
P_{2 k}(x, 0,1,0, y, 1)=T_{2 k}^{A}(x, y) \quad \text { and } \quad P_{2 k+1}(x, 0,1,0, y, 1)=0,
$$

where $k$ is a nonnegative integer. By setting $z, v \rightarrow 1$ and $y, w \rightarrow 0$ and $u \rightarrow y$ in Theorem 1.5, using formula (4.9), we obtain that

$$
\sum_{k=0}^{\infty} T_{2 k}^{A}(x, y) \frac{t^{2 k}}{(2 k) !}=\frac{e^{\frac{x-y}{2} t^{2}}}{{ }_{1} F_{1}\left(-\frac{y}{2(x-y)} ; \frac{1}{2} ; \frac{x-y}{2} t^{2}\right)}
$$

For the case that $n \geq 3$ is odd, we set $z, v \rightarrow 1$ and $y, w \rightarrow 0$ and $u \rightarrow y$ in (1.10). Notice that only the alternating permutations with odd length are not vanished in this case. By the labeling rules in proof of Theorem 2.2, we have

$$
Q_{2 k+1}(x, 0,1,0, y, 1) / y=T_{2 k+1}^{A}(x, y) \quad \text { and } \quad Q_{2 k}(x, 0,1,0, y, 1) / y=0,
$$

where $k$ is a positive integer. By letting $z, v \rightarrow 1$ and $y, w \rightarrow 0$ and $u \rightarrow y$ in Theorem 1.9, then using formula

$$
(1+a-b)_{1} F_{1}(a ; b ; z)=a_{1} F_{1}(a+1 ; b ; z)+(1-b)_{1} F_{1}(a ; b-1 ; z),
$$

we derive that

$$
\sum_{k=1}^{\infty} T_{2 k+1}^{A}(x, y) \frac{t^{2 k+1}}{(2 k+1) !}=\frac{t e^{\frac{x-y}{2} t^{2}}{ }_{1} F_{1}\left(\frac{x}{2(x-y)} ; \frac{3}{2} ;-\frac{x-y}{2} t^{2}\right)}{{ }_{1} F_{1}\left(-\frac{y}{2(x-y)} ; \frac{1}{2} ; \frac{x-y}{2} t^{2}\right)}-1
$$

Therefore, summing $T_{1}^{A}(x, y)=1$ and (4.19), (4.20) together yields (1.11), which completes the proof.

Acknowledgements. The authors appreciate Professor William Y.C. Chen for the valuable suggestions.

\section{References}

[1] M. Abramowitz and I.A. Stegun, Handbook of mathematical functions: with formulas, graphs, and mathematical tables. Vol. 55. Courier Corporation, 1964.

[2] M. Aguiar, N. Bergeron and K. Nyman, The peak algebra and the descent algebra of type B and D, Trans. Amer. Math. Soc. 356 (2004), 2781-2824.

[3] P. Barry, Constructing exponential Riordan arrays from their $A$ and $Z$ sequences, $J$. Integer Seq. 17 (2014), 14.2.6.

[4] N. Basset, Counting and generating permutations using timed languages, Latin American Symposium on Theoretical Informatics, Springer, Berlin, Heidelberg, 2014, 502-513. 
[5] L. Carlitz and R. Scoville, Generalized Eulerian numbers: combinatorial applications, J. Reine Angew. Math. 265 (1974), 110-137.

[6] W.Y.C. Chen, Context-free grammars, differential operators and formal power series, Theoret. Comput. Sci. 117 (1993), 113-129.

[7] W.Y.C. Chen and A.M. Fu, Context-free grammars for permutations and increasing trees, Adv. in Appl. Math. 82 (2017),58-82.

[8] D. Dumont, Grammaires de Willam Chen et dérivations dans les arbres et arborescences, Sém. Lothar. Combin. 37 (1996), B37a, 21 pp.

[9] S. Elizalde and M. Noy, Consecutive patterns in permutations, Adv. in Appl. Math. 20 (2003), 110-125.

[10] A.M. Fu, A context-free grammar for peaks and double descents of permutations, Adv. in Appl. Math. 100 (2018), 179-196.

[11] I.P. Goulden and D.M. Jackson, Combinatorial Enumeration, Courier Corporation, 2004.

[12] S. Kitaev, Generalized pattern avoidance with additional restrictions, Sém. Lothar. Combin. 48 (2003), B48e, 19 pp.

[13] S. Kitaev, Partially ordered generalized patterns, Discrete Math. 298 (2005), 212-229.

[14] S.-M. Ma, Derivative polynomials and enumeration of permutations by number of interior and left peaks, Discrete Math. 312 (2012), 405-412.

[15] R.P. Stanley, Enumerative Combinatorics, Volume I (Second Edition), Cambridge University Press, 2012.

[16] G.N. Watson, A theory of asymptotic series, Philos. Trans. Roy. Soc. London, A 211 (1911), 279-313.

[17] E.T. Whittaker, On the functions associated with the parabolic cylinder in harmonic analysis, Proc. Lond. Math. Soc. (3), 1 (1902), 417-427.

[18] E.T. Whittaker and G.N. Watson, A Course in Modern Analysis, 4th ed., Cambridge University Press, 1952.

[19] ParabolicCylinderD, Wolfram Research, http://functions. wolfram.com/PDF/ParabolicCylinderD.pdf

[20] The On-Line Encyclopedia of Integer Sequences, https://oeis.org/. 